DOCUMENTS

pour l'histoire

des techniques
Documents pour l'histoire des techniques

Nouvelle série

$15 \mid 1^{\text {er }}$ semestre 2008

Minorités et circulations techniques du Moyen-Âge à l'époque Moderne

Michèle Moyne, Catherine Guillot, Jacques Foucart, $D u$ dessin au vitrail. Peintres et maîtres verriers du XIX siècle au nord de la France (Palais des Beaux-Arts de Lille, 8 avril 2006 au 3 juillet 2006) Lille, Palais des Beaux-Arts, 2006, 80 pages, ill. en noir et en couleurs.

Martine Mille

\title{
OpenEdition
}

Journals

Édition électronique

URL : http://journals.openedition.org/dht/1038

DOI : $10.4000 /$ dht. 1038

ISSN : 1775-4194

Éditeur :

Centre d'histoire des techniques et de l'environnement du Cnam (CDHTE-Cnam), Société des élèves du CDHTE-Cnam

Édition imprimée

Date de publication : 1 juin 2008

Pagination : 252-254

ISBN : 978-2-95-30779-1-9

ISSN : 0417-8726

Référence électronique

Martine Mille, « Michèle Moyne, Catherine Guillot, Jacques Foucart, Du dessin au vitrail. Peintres et maitres verriers du xix ${ }^{e}$ siècle au nord de la France (Palais des Beaux-Arts de Lille, 8 avril 2006 au 3 juillet 2006) », Documents pour l'histoire des techniques [En ligne], 15 | $1^{\mathrm{er}}$ semestre 2008, mis en ligne le 21 octobre 2010, consulté le 23 septembre 2020. URL : http://journals.openedition.org/dht/1038; DOI : https://doi.org/10.4000/dht.1038 


\section{Michèle Moyne, Catherine Guillot, Jacques Foucart, Du dessin au vitrail. Peintres et maîtres verriers du XIX siècle au nord de la France (Palais des Beaux-Arts de Lille, 8 avril 2006 au 3 juillet 2006)}

Lille, Palais des Beaux-Arts, 2006, 80 pages, ill. en noir et en couleurs. Martine Mille

\section{RÉFÉRENCE}

Michèle Moyne, Catherine Guillot, Jacques Foucart, Du dessin au vitrail. Peintres et maîtres verriers du XIX ${ }^{e}$ siècle au nord de la France (Palais des Beaux-Arts de Lille, 8 avril 2006 au 3 juillet 2006), Lille, Palais des Beaux-Arts, 2006, 80 pages, ill. en noir et en couleurs.

1 Les dessins préparatoires de peintres du Nord, destinés aux vitraux des édifices religieux de Lille et alentours et représentatifs du style en vogue au milieu du XIX ${ }^{\mathrm{e}}$ siècle, sont l'objet du catalogue Du dessin au vitrail. Peintres et maîtres verriers du XIXe siècle au nord de la France. Présenté par Michèle Moyne, commissaire de l'exposition, le volume réunit une soixantaine d'œuvres retrouvées dans les collections du Palais des Beaux-arts de Lille et restituées au public par l'exposition éponyme de 2006. Grâce à une "réduction en plaquette précieuse", les auteurs réussissent à représenter le gigantisme des cartons de peintres, exécutés au fusain et au crayon noir pour les maîtres verriers, ainsi que les vitraux longilignes de trois à cinq mètres de haut réalisés au milieu du XIX ${ }^{\mathrm{e}}$ siècle, visant à élever l'âme du fidèle et susciter l'émotion religieuse, 
avant le foisonnement des arts décoratifs dans l'espace public et le Modern-style du second XIX ${ }^{\mathrm{e}}$ siècle.

2 En février 2004, le récolement des dessins du XIX ${ }^{\mathrm{e}}$ siècle conservés au Palais des BeauxArts de Lille, comme le précise Jacques Foucart, conservateur général honoraire du Département des peintures du Musée du Louvre, révélait dans les fonds du musée plusieurs cartons de vitraux dont certains liés aux verrières décorant actuellement des églises de Lille. Au printemps 2006, l'exposition du Palais des Beaux-arts Du dessin au vitrail. Peintres et maitres verriers $d u X_{X}{ }^{e}$ siècle au nord de la France constituait une étape importante dans la compréhension des liens entre peintres et maîtres verriers au XIX ${ }^{\mathrm{e}}$ siècle, de la première esquisse, aux dessins et à la réalisation finale du vitrail, présentant également les reproductions photographiques des verrières encore en place aujourd'hui dans les églises lilloises.

3 En relation avec l'exposition du Palais des Beaux-arts de Lille de 2006, le catalogue s'intéresse au renouveau que connaît l'art du vitrail dans le Nord de la France au XIX ${ }^{e}$ siècle. Les œuvres restituées en un volume broché au riche papier glacé rappellent la mise en valeur lors de l'exposition des cartons et dessins jamais présentés au public depuis leur acquisition par la ville de Lille en 1870 grâce au peintre et collectionneur Camille Benoit. Exécutés par deux artistes, Victor Mottez (1809-1897), élève d'Ingres, et Bruno Chérier (1816-1880), ami de Carpeaux, directeur de l'école de dessin de Tourcoing, ces études préparatoires pour des verrières, de taille imposante parfois, s'avèrent être des chefs-d'œuvre à part entière et révèlent un aspect méconnu de la fabrication du vitrail. Elles peuvent désormais être appréhendées comme un patrimoine pictural encore à découvrir.

4 Le catalogue permet de resituer les arts décoratifs religieux du Nord de la France dans l'histoire du vitrail au XIX ${ }^{e}$ siècle. On apprend que la Manufacture de Sèvres a joué un rôle majeur, sous l'impulsion d'Alexandre Brongniart à partir des cartons-chefsd'œuvre d'Ingres destinés aux églises parisiennes, avec des verrières aux larges aplats de couleurs d'une qualité inégalable, référence des arts du feu. Charles Gaudelet, le maître-verrier avec qui travailla exclusivement Mottez et occasionnellement Chérier, était jusqu'à aujourd'hui quasiment inconnu. Les recherches menées tant dans les collections du Palais des Beaux-arts de Lille, que dans les archives conservées dans les fonds anciens de la Bibliothèque de la ville, en relation avec le service régional de l'inventaire et menée par Catherine Guillot, conservateur du patrimoine à la DRAC, sont mises en perspective, et attestent de l'interdisciplinarité des institutions dans la valorisation du patrimoine régional, pictural, manuscrit, architectural ou décoratif du $\mathrm{XIX}^{\mathrm{e}}$ siècle. Ce catalogue, par une lecture dynamique suggère au lecteur de partir à la découverte des édifices religieux aux vitraux si heureusement documentés, parcours qui déborde largement la cité pour proposer la découverte de vitraux d'églises de Flandres de part et d'autre de la frontière, souvent requalifiés grâce aux recherches dans les collections lilloises.

5 L'ouvrage mise sur la sobriété de la présentation et l'heureuse correspondance, en pleine page et en couleur, des vitraux associés aux cartons des peintres, d'une grande pureté. Le lecteur peut saisir, par le trait, la dynamique et l'élan de l'ornementation religieuse. C'est ainsi un « dialogue pictural » que propose ce catalogue. Michèle Moyne restitue les relations privilégiées, continues et attestées de l'artiste religieux et élève d'Ingres, Victor Mottez, avec Feckedey (son agent sur place en réalité), également avec le maître verrier du Nord, Charles Gaudelet, relation perdue parce qu'occultée par le 
peintre, les vitraux portant la signature du seul « faiseur » de Mottez. On perçoit ici la distance entre le parisianisme de l'élève d'Ingres et l'exécution en art par l'artisan nordiste, le vitrail relevant plus de la technique ou du savoir-faire, avec les contraintes des matériaux. Se dessine ainsi une collaboration artistique de vingt ans, née d'une heureuse pérégrination dans les églises parisiennes en 1845 , au service d'un idéal religieux aux commanditaires souvent exigeants, l'un, le peintre, misant sur la force du trait dans ses compositions, l'autre, le verrier, se jouant des contraintes de l'art du feu et de ses supports, tous deux magnifiant la lumière dans les églises. Le catalogue permet de comprendre le décalage entre la composition du peintre-cartonnier et le rendu des verrières. Les cartons de grande qualité sont souvent pleins de promesses, mais le support de verre semble trahir l'artiste. En effet, les contraintes sont multiples qui dénaturent l'élan de l'artiste et la rigueur majestueuse de la liturgie : une peinture immatérielle sans fond, un verre où le jeu des couleurs semble voué au verrier alors que le peintre mentionne rarement la mise en couleur pour ses cartons, des ornementations souvent lourdes destinées à encadrer les sujets, des panneaux insérés entre d'utilitaires plombs de soudure.

6 Les relations de Charles Gaudelet avec le Valenciennois Bruno Chérier, modèle et ami de Jean-Baptiste Carpeaux, et dont la carrière de peintre-décorateur religieux dans le Nord débute en 1852, sont également restituées dans le catalogue par Catherine Guillot. En effet, dans les fonds lillois, certains cartons attribués à Chérier attestent d'un apport au vitrail régional plus important que supposé de prime abord. Chérier travaille peu de temps avec les ateliers Gaudelet mais cette collaboration est établie par la comparaison des verrières encore en place avec les cartons du musée de Lille. Dans ses cartons, Chérier reprend des modèles d'Ingres, fortement influencé par Mottez, mais verse comme cartonnier dans le décalque ou la répétition, tel un « artisan » face au magistral élève d'Ingres, sous influence donc, même si ses compositions donnent à voir des anges tirés de la mystique médiévale, nimbés d'ornementation Renaissance, se rapprochant alors de Viollet-le-Duc.

7 Enfin, les sources d'archives permettent de comprendre la place des verriers dans la cité. Souvent recrutés par leurs commanditaires pour la restauration de verrières, perçus alors comme des " rénovateurs en art ancien », leur rôle de créateur est souvent occulté. Les liens avec l'architecte sont précisés : des tandems sont pointés, souvent originaires de Valenciennes, avec Bruno Chérier. Les cartons "parlent " lors de la restauration, sur papier solide, circonscrits aux délimitations de formes des pièces de verre, les barlotières figurées dans le dessin, témoins du travail d'atelier et éclairant la collaboration étroite entre cartonnier et verrier. Les commandes concrétisent en ce XIX ${ }^{e}$ siècle une réelle chaîne décisionnelle, opérationnelle, artistique et financière au service des arts décoratifs : entre l'architecte, souvent édile de la cité, la fabrique et la cure, des familles d'industriels intervenants alors comme financiers par des visées paternalistes de notables, le peintre cartonnier introduit dans les milieux artistiques parisiens et ses réseaux de sociabilités en région, d'écoles en société de beaux-arts, ses agents souvent peintres comme lui, véritables intermédiaires avec les verriers, considérés en bout de chaîne, au mieux comme des « faiseurs d'art » ou des « artisans " contraints par les matériaux et la technique.

8 Outre le regard inédit qu'il propose sur le travail de ces peintres dans le Nord, le catalogue aborde, fait assez exceptionnel, la spécificité des restaurations entreprises en 2005 sur les dessins en vue de leur présentation et analyse les problématiques 
muséographiques envisagées pour exposer ce type d'œuvres. En effet, la conservation d'œuvres de grand format n'est pas aisée : souvent le roulage nécessaire provoque plis et cassures, tâches ou déchirures. La Manufacture de Sèvres a choisi pour les grandes huiles sur toiles, redécouvertes abandonnées dans ses greniers, de les consolider en les marouflant sur un support rigide, les rendant aisément accrochables ensuite comme des tableaux, cela dans le respect des œuvres sans intervention esthétique.

Les 85 cartons de Mottez et Chérier, conservés au Palais des Beaux-arts de Lille, inventoriés, considérés comme perdus et heureusement retrouvés, roulés et stockés verticalement dans les caves, soumis à la poussière et l'humidité, ont ainsi fait l'objet de méthodes japonaises de restauration visant d'une part à l'exposition temporaire au public, d'autre part et surtout à une conservation appropriée dans des collections ainsi réhabilitées. Chaque pièce a été dépoussiérée, les déchirures consolidées par le verso, par de fines bandes de papier japonais, encollées d'amidon, pour permettre de retrouver la planéité, pour une manipulation aisée, opération accompagnée d'un doublage général par de grandes feuilles et destiné au montage sur des cartons de présentation.

L'architecte Christophe Dumont, choisissant de miser par le jeu des pleins et des vides, avait proposé par une saisissante reconstruction scénographique, d'amener le visiteur dans des salles voulues dans l'esprit des édifices religieux, de manière à préserver le caractère monumental des œuvres, montrées verticalement et en hauteur, et évoquant les vitraux définitifs dans une mise en scène élégante. Ainsi, les cartons de peintres cartonniers du XIX ${ }^{e}$ siècle ont-ils pu être présentés au public en 2006, lors de l'exposition temporaire, heureuse issue d'une lourde mais incontournable campagne de restauration que restitue le catalogue $D u$ dessin au vitrail. Peintres et maitres verriers $d u$ XIXe siècle au nord de la France, dans cette approche patrimoniale spécifique aux grands formats du Nord de la France.

\section{AUTEURS}

\section{MARTINE MILLE}

CDHTE-Cnam 